Article

\title{
High Prevalence and Diversity of Cephalosporin-Resistant Enterobacteriaceae Including Extraintestinal Pathogenic E. coli CC648 Lineage in Rural and Urban Dogs in Northwest Spain
}

\author{
Fátima Abreu-Salinas ${ }^{1,2}$, Dafne Díaz-Jiménez ${ }^{3,4}$, Isidro García-Meniño ${ }^{3,4} \oplus$, Pilar Lumbreras ${ }^{1,2}$, \\ Ana María López-Beceiro ${ }^{5}{ }^{(0)}$, Luis Eusebio Fidalgo ${ }^{5}$, María Rosario Rodicio ${ }^{2,6}$, \\ Azucena Mora ${ }^{3,4,+}$ (D) and Javier Fernández ${ }^{1,2, *,+}$ \\ 1 Servicio de Microbiología, Hospital Universitario Central de Asturias, 33011 Oviedo, Spain; \\ fatimabreu2688@gmail.com (F.A.-S.); pilar.lumbreras08@gmail.com (P.L.) \\ 2 Grupo de Microbiología Traslacional, Instituto de Investigación Sanitaria del Principado de Asturias (ISPA), \\ 33011 Oviedo, Spain; rrodicio@uniovi.es \\ 3 Laboratorio de Referencia de Escherichia coli (LREC), Dpto. de Microbioloxía e Parasitoloxía, \\ Universidade de Santiago de Compostela (USC), 15705 Lugo, Spain; dafne.diaz@usc.es (D.D.-J.); \\ isidro.garcia@usc.es (I.G.-M.); azucena.mora@usc.es (A.M.) \\ 4 Instituto de Investigación Sanitaria de Santiago de Compostela (IDIS), 15706 Santiago, Spain \\ 5 Dpto. de Anatomía, Produción Animal e Ciencias Clínicas Veterinarias, Universidade de Santiago de \\ Compostela (USC), 15705 Lugo, Spain; anam.lopez.beceiro@usc.es (A.M.L.-B.); luis.fidalgo@usc.es (L.E.F.) \\ 6 Departamento de Biología Funcional (Área de Microbiología), Universidad de Oviedo, 33003 Oviedo, Spain \\ * Correspondence: javifdom@gmail.com \\ + These authors contributed equally to this paper.
}

Received: 13 July 2020; Accepted: 30 July 2020; Published: 1 August 2020

\begin{abstract}
The aim of this work was to assess the prevalence of extended spectrum- $\beta$-lactamase (ESBL)and carbapenemase-producing Enterobacteriaceae in fecal samples recovered from rural and urban healthy dogs in Northwest Spain (Galicia) to identify potential high-risk clones and to molecularly characterize positive isolates regarding the genes coding for ESBL/pAmpC resistance and virulence. Thirty-five (19.6\%) out of 179 dogs were positive for cephalosporin-resistant Enterobacteriaceae, including Escherichia coli and Klebsiella pneumoniae (39 and three isolates, respectively). All the isolates were multidrug resistant, with high rates of resistance to different drugs, including ciprofloxacin (71.4\%). A wide diversity of ESBL/pAmpC enzymes, as well as E. coli phylogroups (A, B1, C, D, E, F and clade I) were found. The eight isolates (20.5\%) found to conform to the ExPEC status, belonged to clones O1:H45-clade I-ST770 (CH11-552), O18:H11-A-ST93-CC168 (CH11-neg), O23:H16-B1-ST453-CC86 (CH6-31), and O83:H42-F-ST1485-CC648 (CH231-58), with the latter also complying the uropathogenic (UPEC) status. The three K. pneumoniae recovered produced CTX-M-15 and belonged to the ST307, a clone previously reported in human clinical isolates. Our study highlights the potential role of both rural and urban dogs as a reservoir of high-risk Enterobacteriaceae clones, such as the CC648 of E. coli and antimicrobial resistance traits. Within a One-Health approach, their surveillance should be a priority in the fight against antimicrobial resistance.
\end{abstract}

Keywords: ST1485 (CC648); ExPEC; MDR; ESBL; AmpC; dogs; E. coli; K. pneumoniae

\section{Introduction}

The increase of antibiotic resistance represents a global threat to human and animal health, being that therapeutic options to combat infections have drastically reduced in recent years [1,2]. According to 
the list of antibiotic-resistant "priority pathogens" of the World Health Organization (WHO), multidrug-resistant (MDR) Enterobacteriaceae, and specifically those producing extended-spectrum $\beta$-lactamases (ESBL) and/or resistant to carbapenems, are critical pathogens in hospitals [2]. Within the Enterobacteriaceae family, Klebsiella pneumoniae and Escherichia coli are currently among the most important clinical burdens for human and animal health, since they have developed resistance against antibiotics regarded as the last line of defense against MDR bacteria [1]. E. coli is a common member of the intestinal microbiota of humans and other mammals, including dogs. However, E. coli can also act as a pathogen causing a wide range of infections from enteric to extraintestinal diseases, which defines the two main pathogenic categories: diarrheagenic E. coli (DEC) and extraintestinal pathogenic E. coli (ExPEC) [3,4]. ExPEC include a heterogeneous group defined by isolation from locations outside the intestinal tract with no set of genes able to unequivocally distinguish them from commensal E. coli: avian pathogenic E. coli (APEC), neonatal meningitis E. coli (NMEC), and uropathogenic E. coli (UPEC) [3,5]. In fact, they can colonize the intestinal tract, which in turn can serve as reservoir [5]. It is particularly within the group of ExPEC, where the successful high-risk clones of E. coli emerge [6]. The constant antibiotic selective pressure in human and animal health may contribute to the selection and further transmission of antibiotic-resistant bacteria from animals to humans and vice versa $[7,8]$. Considering that the number of humans having pets is increasing worldwide, companion animals may play an important role in the dissemination of MDR bacteria to their owners [9-11]. Currently, numerous studies report on the colonization and infection of companion animals by MDR bacteria, including ESBL- and carbapenemase-producing Enterobacteriaceae of a wide variety of species and clones, with a wide range of enzymes [10-14]. These bacteria may be acquired via different routes, such as direct contact with humans, feeding with raw food products, and outdoor living or interacting [15-17].

Institutions, such as the World Health Organization (WHO), the European Centre for Disease Prevention and Control (ECDC), and the Centers for Disease Control and Prevention (CDC), urge an effort to implement a "One-Health" approach involving human and veterinary health collaboration to fight against antibiotic resistance [18,19]. For this reason, the aim of this work was to assess the prevalence of ESBL- and carbapenemase-producing Enterobacteriaceae in fecal samples of rural and urban healthy dogs in Northwest Spain (Galicia), to identify potential high-risk clones for humans and to molecularly characterize positive isolates regarding the genes coding for ESBL/pAmpC (plasmid-mediated AmpC-beta-lactamases) resistance and virulence.

\section{Results}

Thirty-five (19.6\%) of the 179 dogs tested carried cephalosporin-resistant Enterobacteriaceae (10 from urban and 25 from rural environments, $20.8 \%$ and $19.1 \%$, respectively). From the 35 positive dogs, 39 ESBL- and/or pAmpC-producing E. coli and three K. pneumoniae ESBL-producing isolates were recovered. Six dogs (14.3\%) carried more than one ESBL- and/or AmpC-producing Enterobacteriaceae (Table S1): E. coli and K. pneumoniae (three dogs), or two and three different E. coli isolates (two and a single dog, respectively).

All 42 isolates were MDR, defined as non-susceptible to at least one agent in three or more antimicrobial categories according to the Magiorakos criteria [20]. Specifically, all isolates $(100 \%)$ were resistant to ampicillin, cefuroxime, and cefotaxime; 30 to ciprofloxacin $(71.4 \%) ; 22$ to trimethoprim/sulfamethoxazole (52.4\%); 15 to tobramycin (35.7\%); 13 to gentamycin $(31.0 \%) ; 12$ to amoxicillin/clavulanic acid (28.6\%); six to amikacin (14.3\%); and a single one to piperacillin/tazobactam $(2.4 \%)$. Resistance to carbapenems, tigecycline, and colistin was not detected, and all isolates were negative for the $m o r$ genes ( 1 to 5 ) analyzed.

Among the 36 ESBL E. coli producers, 27 (75\%) carried a bla $a_{\mathrm{CTX}-\mathrm{M}}$ gene: $b l a_{\mathrm{CTX}-\mathrm{M}-1}(6), b l a_{\mathrm{CTX}-\mathrm{M}-14}(6)$, $b l a_{\mathrm{CTX}-\mathrm{M}-15}(5), b l a_{\mathrm{CTX}-\mathrm{M}-32}(4), b l a_{\mathrm{CTX}-\mathrm{M}-65}$ (3), bla $a_{\mathrm{CTX}-\mathrm{M}-27}$ (2), bla $a_{\mathrm{CTX}-\mathrm{M}-55}$ (1), while nine (25\%) were positive for $b l a_{\mathrm{SHV}-12}$. In addition, eight $E$. coli, each from a different dog, were positive for a pAmpC-encoding gene $b l a_{\mathrm{CMY}-2-\text { like }}: b l a_{\mathrm{CMY}-171}$ (5), bla $a_{\mathrm{CMY}-42}$ (2) and $b l a_{\mathrm{CMY}-2}$ (1). The phylogenetic analysis of the 39 broad-spectrum cephalosporin-resistant $E$. coli (ESBL/pAmpC producers) revealed a 
high heterogeneity, with isolate belonging to six phylogroups sensu stricto (A, B1, C, D, E and F) and clade I. The phylogroup B1 was the most prevalent (14 isolates; 35.9\%), followed by A (7), C (6), clade I (5), E (3), F (3), and D (1). The screening of virulence traits showed that eight (20.5\%) of E. coli isolates conformed the ExPEC status and belonged to four clones (combination of serotype-phylogroup-ST/CC-clonotype): O1:H45-clade I-ST770 (CH116-552) (5 isolates), O18:H11-A-ST93-CC168 (CH11-neg) (1 isolate), O23:H16-B1-ST453-CC86 (CH6-31) (1 isolate), and O83:H42-F-ST1485-CC648 (CH231-58) (1 isolate). The latter, (ST1485-CC648), also complied with the UPEC status. These isolates carried additional extraintestinal virulence traits (Table 1). Of note is the high number of virulence genes (16) of the ST1485-CC648, SHV-12-producing isolate. Pulse field gel electrophoresis (PFGE) performed to all E. coli isolates yielded 30 profiles (Figure 1).

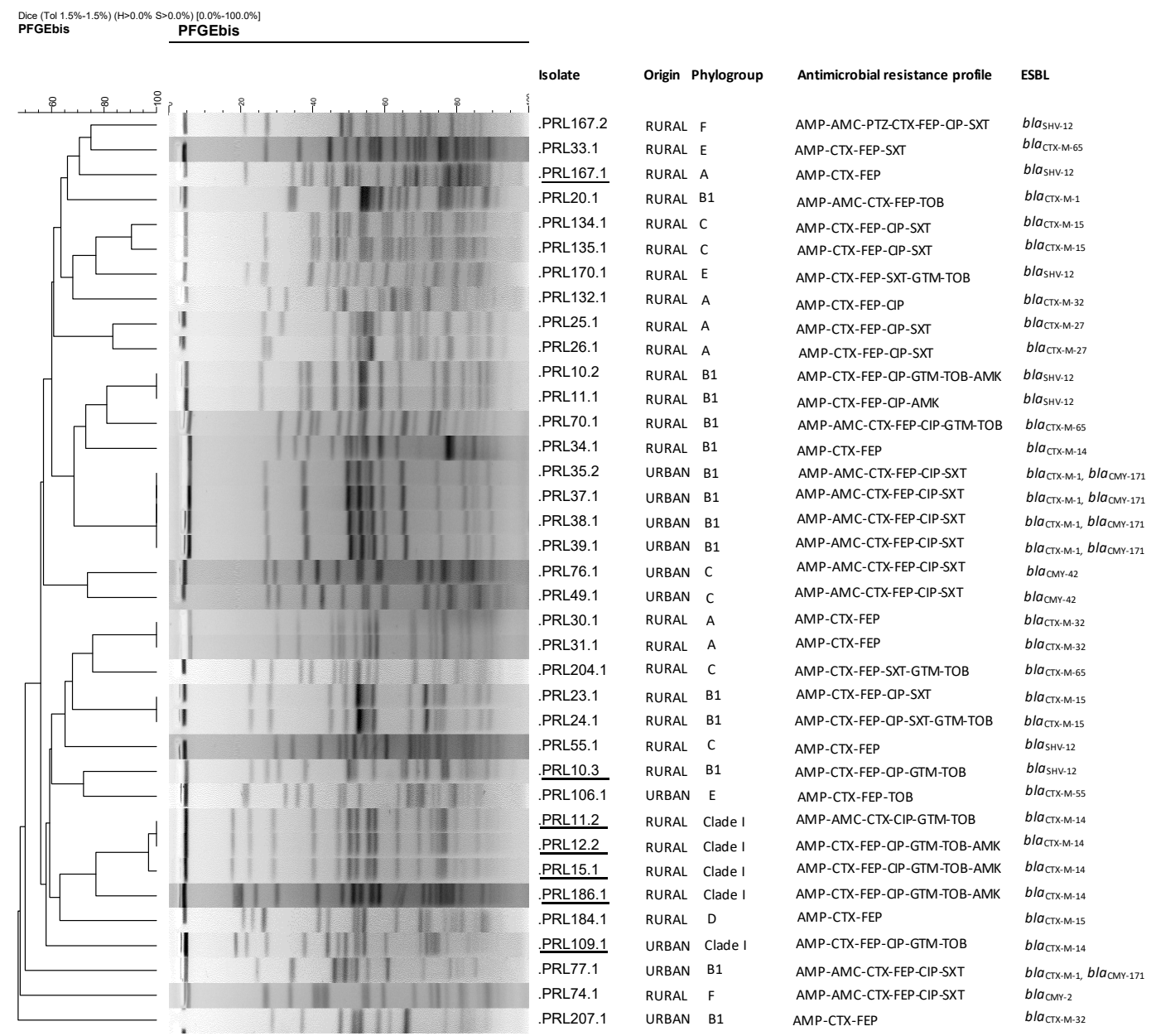

Figure 1. Dendrogram of genomic similarity obtained by XbaI-PFGE typing of ESBL-producing Escherichia coli isolates from rural and urban dogs in Northwest Spain. Isolate numeration was done by using the correlative number of the dog studied, followed by a dot and a second number if the same dog has more than one positive isolate. AMP, ampicillin; AMC, amoxicillin/clavulanic acid; PTZ, piperacillin/tazobactam; CTX, cefotaxime; FEP, cefepime; CIP, ciprofloxacin; SXT, trimethoprim/sulphamethoxazole; GTN, gentamicin; TOB, tobramycin; AMK, amikacin. Isolates conforming ExPEC status are underlined. Two isolates (PRL10.1, which also fulfills the ExPEC status, and PRL19.1) are not shown since they were non-typable by PFGE due to DNA degradation. 
Table 1. Phenotypic and genotypic characterization of the eight E. coli conforming the ExPEC status.

\begin{tabular}{|c|c|c|c|c|c|c|c|}
\hline Isolate & Serotype & PG & ST (CC) & ${ }^{1} \mathrm{CH}$ & ESBL & ${ }^{2}$ Antimicrobial Resistance & ${ }^{3}$ Virulence-Gene Profile \\
\hline PRL11.2 & $\mathrm{O} 1: \mathrm{H} 45$ & clade I & 770 (None) & $116-552$ & CTX-M-14 & AMP-AMC-CTX-CIP-GTM-TOB & fimH552 iucD iutA kpsM II-K5 traT malX chuA \\
\hline PRL12.2 & O1:H45 & clade I & 770 (None) & $116-552$ & CTX-M-14 & AMP-CTX-FEP-CIP-GTM-TOB-AMK & fimH552 hlyF iucD iutA kpsM II-K5 traT malX chuA \\
\hline PRL15.1 & O1:H45 & clade I & 770 (None) & $116-552$ & CTX-M-14 & AMP-CTX-FEP-CIP-GTM-TOB-AMK & fimH552 hlyF iucD iutA kpsM II-K5 traT malX chuA \\
\hline PRL109.1 & $\mathrm{O} 1: \mathrm{H} 45$ & clade I & 770 (None) & $116-552$ & CTX-M-14 & AMP-CTX-FEP-CIP-GTM-TOB & fimH552 hlyF iucD iutA kpsM II-K5 traT malX chuA \\
\hline PRL186.1 & $\mathrm{O} 1: \mathrm{H} 45$ & clade I & 770 (None) & $116-552$ & CTX-M-14 & AMP-CTX-FEP-CIP-GTM-TOB-AMK & fimH552 hlyF iucD iutA kpsM II-K5 traT malX chuA \\
\hline PRL167.1 & O18:H11 & A & $93(168)$ & 11-neg & SHV-12 & AMP-CTX-FEP & hlyF iucD iutA kpsM II-K5 \\
\hline PRL10.3 & $\mathrm{O} 23: \mathrm{H} 16$ & B1 & $453(86)$ & $6-31$ & SHV-12 & AMP-CTX-FEP-CIP-GTM-TOB & fimH31 hlyF iucD iutA iron kpsM II-K5 cvaC traT iss fyuA \\
\hline PRL10.1 & O83:H42 & $\mathrm{F}$ & $1485(648)$ & $231-58$ & SHV-12 & AMP-CTX-FEP-CIP-SXT & $\begin{array}{c}\text { fimH48 hlyF iucD iutA iron kpsM II-K5 cvaC traT malX tsh } \\
\text { ompT iss chuA vat fyuA yfcV }\end{array}$ \\
\hline
\end{tabular}

${ }^{1}$ Clonotype (CH) based on the internal 469-nucleotide (nt) and 489-nt sequence of the fumC (allele obtained from multilocus sequence typing (MLST) and fimH genes, respectively

(Weissman et al., 2012): neg when PCR was negative for the 489-nt internal sequence amplification. ${ }^{2}$ Phenotypic resistance interpreted according to the European Committee on Antimicrobial

Susceptibility testing (EUCAST) guidelines: AMP, ampicillin; AMC, amoxicillin/clavulanic acid; CTX, cefotaxime; FEP, cefepime; CIP, ciprofloxacin; SXT, trimethoprim/sulfamethoxazole;

GTM, gentamicin; TOB, tobramycin; AMK, amikacin. ${ }^{3}$ PRL10.1 isolate complied also with the UPEC status. PG, phylogroup; ST, sequence type; CC, clonal complex. 
The XbaI-PFGE dendrogram corroborated their diversity with some isolates showing less than $60 \%$ similarity. However, six clusters (>85\% identity) grouped individual isolates belonging to the same phylogroup and recovered in the same location (Figure 1; Table S1). Two isolates were non-typable by PFGE due to DNA degradation: PRL19.1 (rural origin; phylogroup A; resistant to AMP, CTX, FEP, CIP, GEN, TOB, AMK; positive for bla $a_{\mathrm{SHV}-12}$; and for iutA) and PRL10.1 (rural origin; phylogroup F; serotype O83:H42; ST1485-CC648 (CH231-58); resistant to AMP, CTX, FEP, CIP, SXT; positive for bla $_{\mathrm{SHV}-12}$ and for iutA, chuA, vat, kpsMII, fulfilling the ExPEC status).

Three ESBL-producing K. pneumoniae were also recovered from three individual dogs (two from urban and one from rural areas). All were positive for the bla $a_{\text {CTX-M-15 }}$ and belonged to the ST307. Interestingly, these K. pneumoniae isolates were found together with pAmpC-producing E. coli (in two dogs) and with one SHV-12 producing E. coli (in one dog).

In summary, we have detected a high rate of fecal colonization by ESBL- and pAmpC-producing Enterobacteriaceae in urban and rural dogs in Galicia, with no statistically significant differences $(20.8 \%$ and $19.1 \%$, respectively).

\section{Discussion}

The rate of colonization by MDR bacteria in companion animals has been assessed in several studies, where the prevalence of colonization by ESBL-producing Enterobacteriaceae among dogs and cats (including healthy and sick animals) ranged widely—between 3.1\% and 55\% [11,12]—while the figures reported only for healthy dogs were around 20\% [12]. These data match the results obtained here, with a prevalence of ca. $20 \%$, regardless of the origin of the dogs (rural or urban). This is also consistent with data from previous studies, which did not find significant differences with respect to the urbanization level of the dogs analyzed [11]. Colonization by ESBL/pAmpC-producing Enterobacteriaceae in companion animals could be related to several factors, including a selective pressure due to previous antibiotic exposure [10] but also to indirect acquisition through raw feeding with meat or carcasses from food-producing animals, which is not uncommon in rural areas. We know that the dogs included in our study had not received any antimicrobial treatment during the previous four weeks before sampling, but, unfortunately, earlier data on antibiotic consumption were not available. A recent study carried out in three European countries (Belgium, Italy, and the Netherlands), found that antimicrobial consumption in companion animals was lower than consumption in food-producing animals. However, the authors reported a high use of WHO critically important antimicrobials, including cefovecin (a third-generation cephalosporin) and quinolones, being that this consumption is higher for dogs than cats [21]. Third and fourth generation cephalosporins, as well as quinolones, have been classified as restricted by the recent categorization of antimicrobials of the European Medicines Agency [22], but they were not prohibited. In a recent survey carried out in Spain, $\beta$-lactams and quinolones were the most prescribed antimicrobials in dogs [23]. It is well known that the use of quinolones typically selects ESBL-producing Enterobacteriaceae [24]. Considering that $71.4 \%$ of the analyzed isolates from our study were resistant to ciprofloxacin, it is tempting to speculate that quinolone exposure in these dogs could have been involved in the selection of ESBL-producing Enterobacteriaceae.

The high genetic diversity and ESBL/pAmpC types found in the present study has been previously described within E. coli recovered from dogs [17,25], as well as the STs 93, 453, and 770, found here in

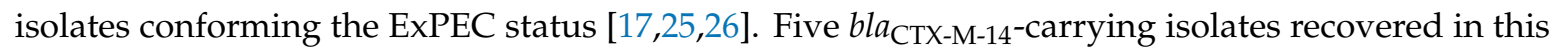
study belonged to ST770 Escherichia clade I. The clade I was considered as a phylogroup of E. coli based on the extent of recombination detected with strains belonging to E. coli sensu stricto [27]. ST770 is an infrequently reported clone, which has been associated with bla $a_{\mathrm{CTX}-\mathrm{M}-1}$ carriage in broilers and poultry in the Netherlands and Switzerland $[28,29]$ and with bla $a_{\mathrm{CTX}-\mathrm{M}-14}$ in a patient diagnosed with a urinary

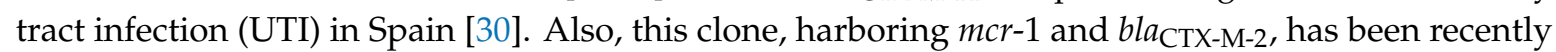
recovered from a dog with a UTI in Argentina [31] and associated with pAmpC production, specifically CMY-2, from rooks wintering in Czechia and from broilers in Sweden [32,33]. 
It is of note that in a recent study performed in our region (Galicia) on chicken and turkey meat, we recovered five (5\%) ESBL-producing Escherichia clade I ST770 (CH116-552) from different samples, all of them positive for the ExPEC status [34]. Furthermore, three of these isolates were O1:H45. In the same study, we recovered ExPEC-positive isolates belonging to the clones O18:H11-A-ST93-CC168 (CH11-neg), O23:H16-B1-ST453-CC86 (CH6-31), and O83:H42-F-ST1485-CC648 (CH231-58). All those isolates from poultry meat were MDR and most of them fluoroquinolone-resistant. As stated above, dogs may acquire antimicrobial resistant Enterobacteriaceae via various routes, including raw feeding with chicken meat or carcasses, which is quite common in rural areas. Poultry products can act as a reservoir for human extraintestinal Enterobacteriaceae pathogens [34-36], so we also hypothesize that such products could be playing a role in their transmission between animals, particularly in rural environments. Importantly, MDR E. coli of human clinical origin and characterized as A-ST93 (CH11-neg), B1-ST453 (CH6-31), and F-ST1485 (CH231-58) were also recently reported in the same health area (Galicia) [37,38]. This reinforces the importance of "One-Health" actions against dissemination of antimicrobial resistance.

Other STs detected in E. coli from dogs in the present study were ST93 and ST453. E. coli ST93 were reported in wild birds in Pakistan, associated with the carriage of blaCTX-M-15 [28]; in beef, veal, pork and poultry, associated with bla $a_{\mathrm{CTX}-\mathrm{M}-1}$ in Switzerland [28]; and in broiler chickens carrying bla in Brazil [39]. In addition, ST93 was also associated to the spread of the mcr-1 gene in companion animals and retail food in China [40,41]. Regarding infections in humans, mcr-1-carrying ST93 E. coli was recovered from a patient with bacteremia in Uruguay [42]. E. coli ST453 harboring bla $\mathrm{CTX}_{\mathrm{M}-\mathrm{M}}$ was isolated from pigs and their breeders [43], associated with extraintestinal disease in humans and metritis in cattle in Australia and, carrying mor-1, with wastewater in Japan [44].

Despite the fact that the pandemic E. coli ST131-B2 was not detected here, eight out of the 39 $(20.5 \%)$ isolates conformed to the ExPEC status, including one isolate of the global ExPEC lineage F-CC648 belonging to the ST1485 [6]. The phylogroup F together with phylogroup B2 comprise most human clinical ExPEC isolates. Among phylogroup F, the clonal complex 648 (CC648) is a resistance-associated lineage recovered from different sources (human, animal, or environmental) and increasingly associated with extraintestinal pathologies [45]. Importantly, the dog isolate also fulfilled the UPEC status, conjugating in the same isolate a high number of resistance and virulence genes.

In addition to $E$. coli isolates, we have found three dogs carrying CTX-M-15-producing K. pneumoniae, which is a major nosocomial pathogen able to persist in many different reservoirs, including not only health care settings but also retail meat, livestock, and wastewater [46,47]. This species belongs to the ESKAPE list and is considered as a pathogen that represents a global threat to human health, especially in hospital environments [1]. Information about ESBL carriage in this species recovered from companion animals, such as dogs, is limited [10]. The ST307 clone of K. pneumoniae found in our study is considered a potential high-risk clone for humans and has been associated with different ESBL- and carbapenemase-encoding genes [48,49]. Recently, it has been obtained from sick and healthy dogs in Vila Real, a city in northern Portugal very close to Galicia, carrying bla $a_{\mathrm{CTX}-\mathrm{M}-15}$ and bla $\mathrm{SHV}_{28}$ [50]. This clone (including CTX-M-15- SHV-28-producing isolates) was also detected in $27 \%$ of the poultry meat samples analyzed by Díaz-Jiménez et al. in Galicia [34].

Fortunately, no carbapenemase-producing Enterobacteriaceae were recovered among the dogs studied. In a previous work, a $0.6 \%$ prevalence of carbapenemase-producing Enterobacteriaceae in the fecal microbiota of companion dogs attending a veterinary hospital in the Community of Madrid (Spain) was reported [13]. Probably because carbapenems are not used in veterinary medicine, bacteria resistant to these drugs are less common than ESBL-producing bacteria in companion animals [14].

The present study has limitations, such as its cross-sectional design, which did not allow subsequent follow up of the dogs studied. Moreover, information about risk factors for MDR bacteria colonization/infection was not available. However, we provide data on the prevalence of ESBL- and pAmpC-producing Enterobacteriaceae among healthy rural and urban dogs in northwest Spain, including extraintestinal pathogenic E. coli lineages, such as CC648, highlighting the potential 
role of these animals in the transmission to humans of high-risk pathogens and resistance genes. Therefore, within a "One-Health" approach, their surveillance should be a priority line in the fight against antimicrobial resistance.

\section{Materials and Methods}

\subsection{Sample Collection, Culture, and Bacterial Identification}

A total of 179 fresh fecal specimens were collected during May and June 2019 from individual healthy dogs living in rural and urban environments in Galicia, a ca. 29,500 $\mathrm{km}^{2}$ region in Northwest Spain. The healthy status of the dogs was established by the veterinary team in charge of the sampling. Sampling was designed to be representative of the entire territory studied. Thus, a total of 43 different geographical areas were screened, selecting dogs from different rural environments of the four provinces of Galicia (A Coruña, Lugo, Ourense, and Pontevedra), as well as from the main cities in the same region. Urban refers to dogs that live in flats with their owners in large or medium-sized towns in Galicia. Their function is as a companion animal (pets) and they do not contact with livestock. In contrast, rural refers to dogs which usually live in rural areas, in smaller towns or villages. But the most important is that the latter are used as guard dogs in farms, or for hunting. Most of these animals are in contact with livestock (poultry, ruminants -bovine and ovine- and porcine) and with wildlife. The sampling size was calculated based on the dog population in Galicia, which according to official data for 2019 (Galician Registry of Identification of Companion Animals, Department of the Environment of the Xunta de Galicia, Spain) is 609,804 dogs. Of these, 147,284 (24.2\%) are urban dogs and 462,520 (75.8\%) are rural dogs. In the present study, we have sampled 179 dogs, of which 48 are urban (26.8\%) and 131 rural (73.2\%) [Table S1]. The rural vs. urban proportions of this work were adjusted to the values of the geographic area. In order to avoid biases, in those cases in which the same owner had several dogs or several dogs lived together in the same area, a single sample of a representative individual was collected. Dogs included in the study had not received any antimicrobial treatment during the previous four weeks. Samples were kept refrigerated $\left(4^{\circ} \mathrm{C}\right)$ in sterile swabs until processing in the laboratory within $24 \mathrm{~h}$ after sampling. For this, they were plated on Chromagar ESBL (bioMérieux, Marcy l'Étoile, France), Chromid Carba Smart (bioMérieux), Chromagar OXA-48 (bioMérieux), and also on Columbia agar with 5\% sheep blood (bioMérieux) used as a growth control. Bacterial isolates growing in selective media were identified by matrix-assisted laser desorption/ionization time-of-flight mass spectrometry (MALDI-TOF/MS, Bruker Daltronics GmbH, Bremen, Germany).

\subsection{Antimicrobial Susceptibility Testing and Characterization of Antimicrobial Resistance-Encoding Genes}

Antimicrobial susceptibility testing of suspicious enterobacterial colonies growing in selective media was performed by the MicroScan WalkAway system (Beckman Coulter, CA, USA), and the results were interpreted according to the EUCAST 2020 breakpoints [51]. The antibiotics tested included: ampicillin, amoxicillin/clavulanic acid, cefotaxime, cefepime, piperacillin/tazobactam, imipenem, meropenem, ciprofloxacin, trimethoprim/sulfamethoxazole, gentamicin, tobramycin, amikacin, colistin, and tigecycline.

All isolates were tested for ESBL-encoding genes $\left(b l a_{\mathrm{TEM}}, b l a_{\mathrm{SHV}}\right.$, and $\left.b l a_{\mathrm{CTX}-\mathrm{M}}\right)$ and for $\mathrm{pAmpCs}$ by PCR amplification followed by sequencing of the positive amplicons using specific primers [Table S2]. Genes encoding plasmid-mediated colistin resistance ( $m c r-1$ to $m c r-5)$ were also screened as previously described [52].

\subsection{Characterization of E. coli Isolates: Virulence Traits, Phylogroups, STs and Clonotypes, Serotyping, and PFGE}

All E. coli were analyzed by PCR for specific virulence markers, which define the ExPEC status and the UPEC status. Isolates conformed the ExPEC status if they were positive for $\geq$ two of five determinants, including papAH and/or papC, sfa/focDE, afa/draBC, kpsM II, and iutA [53], and met 
the UPEC status if positive for $\geq$ three of the four genes, including $\operatorname{chu} A, f y u A$, vat, and $y f_{c} V$ [54]. Those isolates exhibiting ExPEC and/or UPEC status were also characterized for other extraintestinal virulence factors: fim $A v_{M T 78}$, papEF, papC, cnf1, cdtB, sat, hlyA, hlyF, iucD, iroN, kpsM II (establishing neuC-K1, K2, and $\mathrm{K} 5$ variants), kpsM III, cvaC, iss, traT, ibeA, malX, usp, tsh, and ompT [Table S3].

The phylogroup of E. coli isolates was determined following the scheme of Clermont et al. [27] [Table S4]. Isolates with ExPEC and/or UPEC status were further characterized for their serotypes, clonotypes and STs. Serotyping was established using the method previously described by Guinee et al. [55] with antisera against $\mathrm{O}(\mathrm{O} 1$ to $\mathrm{O} 185)$ and $\mathrm{H}$ (H1 to H56) antigens. Clonotyping was accomplished by sequencing 469 nucleotides (nt) internal to the fum $\mathrm{C}$ gene and $489 \mathrm{nt}$ internal to fimH, which allowed us to define the CH type [56] [Table S5]. ST assignment for E. coli and for K. pneumoniae isolates was performed according to the Achtman and the Diancourt MLST schemes, respectively [57,58] [Table S6 and Table S7].

Pulsed-field gel electrophoresis (PFGE) was performed to E. coli isolates as previously described using XbaI [34], and the profiles obtained were compared and analyzed by InfoQuest ${ }^{\mathrm{TM} F P}$ v.4.5 software (Bio-Rad Laboratories). A dendrogram was constructed by the UPGMA (Unweighted Pair Group Method with Arithmetic Mean) method, based on Dice's similarity coefficient (1.5\% band tolerance; $1.5 \%$ optimization).

\subsection{Statistical Analysis}

Differences in colonization between urban and rural dogs were analyzed by a two-tailed Fisher's exact test, with $\mathrm{p}$ values of less than 0.05 being considered as statistically significant.

\section{Conclusions}

Our study highlights the potential role of both rural and urban dogs as a reservoir of high-risk Enterobacteriaceae clones, such as the CC648 of E. coli and antimicrobial resistance traits. Within a One-Health approach, their surveillance should be a priority in the fight against antimicrobial resistance.

Supplementary Materials: The following are available online at http://www.mdpi.com/2079-6382/9/8/468/s1, Table S1: dog sampling data; Table S2: primers used for the detection and/or sequencing of $b l a_{\mathrm{CTX}-\mathrm{M}}, b l a_{\mathrm{SHV}}$, $b l a_{\mathrm{TEM}}, b l a_{\mathrm{CMY}}$, and mcr genes; Table S3: targets and primers associated with extraintestinal pathogenic E. coli; Table S4: targets and primers to determine phylogroups of E. coli (Clermont et al., 2013); Table S5: targets and primers used to determine clonotypes; Table S6: targets and primers to determine sequence types by MLST (E. coli), Table S7: targets and primers to determine sequence types by MLST (K. pneumoniae).

Author Contributions: Conceptualization, M.R.R., A.M. and J.F.; formal analysis, F.A.-S., M.R.R., A.M. and J.F.; funding acquisition, A.M. and J.F.; investigation, F.A.-S., D.D.-J., I.G.-M., P.L., A.M.L.-B. and L.E.F.; supervision, M.R.R., A.M. and J.F.; writing-original draft, J.F.; writing—review and editing, F.A.-S., D.D.-J., I.G.-M., P.L., M.R.R., A.M. and J.F. All authors have read and agreed to the published version of the manuscript.

Funding: This research was funded project FIS PI17-00728 (Fondo de Investigación Sanitaria, Instituto de Salud Carlos III, Ministerio de Economía y Competitividad, Spain), cofunded by the European Regional Development Fund of the European Union: a Way to Making Europe (FEDER); Project PID2019-104439RB-C21/AEI/10.13039/501100011033 and FEDER; ED431C 2017/57 from the Consellería de Cultura, Educación e Ordenación Universitaria, Xunta de Galicia and FEDER; and by the Strategic Researcher Cluster BioReDeS funded by the Regional Government Xunta de Galicia under the project no. ED431E 2018/09. D. Díaz-Jiménez and I. García-Meniño acknowledge the Consellería de Cultura, Educación e Ordenación Universitaria, Xunta de Galicia for their pre-doctoral grants (ED481A-2019/022 and ED481A-2015/149, respectively). The Research stay of I. García-Meniño at the Hospital Universitario Central de Asturias was funded by a grant from the Sociedad Española de Enfermedades Infecciosas y Microbiología Clínica (SEIMC).

Acknowledgments: We thank J.J. Palacios for its valuable help for the dendrogram construction.

Conflicts of Interest: The authors declare no conflict of interest. The funders had no role in the design of the study; in the collection, analyses, or interpretation of data; in the writing of the manuscript, or in the decision to publish the results. 


\section{References}

1. De Oliveira, D.M.P.; Forde, M.B.; Kidd, J.T.; Harris, N.A.P.; Schembri, A.M.; Beatson, A.S.; Paterson, L.D.; Walker, J.M. Antimicrobial resistance in ESKAPE pathogens. Clin. Microbiol. Rev. 2020, 33. [CrossRef]

2. Mo, M. Antibiotic-Resistant Priority Pathogens List. Available online: https://www.who.int/ (accessed on 6 June 2020).

3. Kaper, J.B.; Nataro, J.P.; Mobley, H.L.T. Pathogenic Escherichia coli. Nat. Rev. Microbiol. 2004, 2, $123-140$. [CrossRef]

4. Amadi, V.A.; Hariharan, H.; Amadi, A.O.; Matthew-Belmar, V.; Nicholas-Thomas, R.; Lanza Perea, M.; Carter, K.; Eugene, R.; Kalasi, K.; Alhassan, A.; et al. Antimicrobial resistance patterns of commensal Escherichia coli isolated from feces of non-diarrheic dogs in Grenada, West Indies. Vet. World 2019, 12, 2070-2075. [CrossRef] [PubMed]

5. Köhler, C.D.; Dobrindt, U. What defines extraintestinal pathogenic Escherichia coli? Int. J. Med. Microbiol. 2011, 301, 642-647. [CrossRef] [PubMed]

6. Manges, A.R.; Geum, H.M.; Guo, A.; Edens, T.J.; Fibke, C.D.; Pitout, J.D. Global extraintestinal pathogenic Escherichia coli (Expec) lineages. Clin. Microbiol. Rev. 2019, 32. [CrossRef] [PubMed]

7. Lloyd, D.H. Reservoirs of antimicrobial resistance in pet animals. Clin. Infect. Dis. 2007, 45, S148-S152. [CrossRef] [PubMed]

8. Argudín, M.A.; Deplano, A.; Meghraoui, A.; Dodémont, M.; Heinrichs, A.; Denis, O.; Nonhoff, C.; Roisin, S. Bacteria from animals as a pool of antimicrobial resistance genes. Antibiotics 2017, 6, 12. [CrossRef] [PubMed]

9. Halsby, K.D.; Walsh, A.L.; Campbell, C.; Hewitt, K.; Morgan, D. Healthy animals, healthy people: Zoonosis risk from animal contact in pet shops, a systematic review of the literature. PLoS ONE 2014, 9, e89309. [CrossRef]

10. Pomba, C.; Rantala, M.; Greko, C.; Edward Baptiste, K.; Catry, B.; van Duijkeren, E.; Mateus, A.; Moreno, A.M.; Pyörälä, S.; Ružauskas, M.; et al. Public health risk of antimicrobial resistance transfer from companion animals. J. Antimicrob. Chemother. 2017, 72, 957-968. [CrossRef]

11. Van den Bunt, G.; Fluit, A.C.; Spaninks, M.P.; Timmerman, A.J.; Geurts, Y.; Kant, A.; Scharringa, J.; Mevius, D.; Wagenaar, J.A.; Bonten, M.J.M.; et al. Faecal carriage, risk factors, acquisition and persistence of ESBL-producing Enterobacteriaceae in dogs and cats and co-carriage with humans belonging to the same household. J. Antimicrob. Chemother. 2020, 75, 342-350. [CrossRef]

12. Baede, V.O.; Wagenaar, J.A.; Broens, E.M.; Duim, B.; Dohmen, W.; Nijsse, R.; Timmerman, A.J.; Hordijk, J. Longitudinal study of extended-spectrum- $\beta$-lactamase-and AmpC-producing enterobacteriaceae in household dogs. Antimicrob. Agents Chemother. 2015, 59, 3117-3124. [CrossRef] [PubMed]

13. González-Torralba, J.; Oteo, A.; Asenjo, V.; Bautista, E.F.; Alós, J.I. Survey of carbapenemase-producing Enterobacteriaceae in companion dogs in Madrid, Spain. Antimicrob. Agents Chemother. 2016, 60, $2499-2501$. [CrossRef] [PubMed]

14. Köck, R.; Daniels-Haardt, I.; Becker, K.; Mellmann, A.; Friedrich, A.W.; Mevius, D.; Schwarz, S.; Jurke, A. Carbapenem-resistant Enterobacteriaceae in wildlife, food-producing, and companion animals: A systematic review. Clin. Microbiol. Infect. 2018, 24, 1241-1250. [CrossRef] [PubMed]

15. Ovejero, C.M.; Escudero, J.A.; Thomas-Lopez, D.; Hoefer, A.; Moyano, G.; Montero, N.; Martin-Espada, C.; Gonzalez-Zorn, B. Highly tigecycline-resistant Klebsiella Pneumoniae sequence TYPE 11 (ST11) \& ST147 isolates from companion animals. Antimicrob. Agents Chemother. 2017, 61. [CrossRef]

16. Ljungquist, O.; Ljungquist, D.; Myrenås, M.; Rydén, C.; Finn, M.; Bengtsson, B. Evidence of household transfer of ESBL-/pAmpC-producing Enterobacteriaceae between humans and dogs-a pilot study. Infect. Ecol. Epidemiol. 2016, 6, 31514. [CrossRef]

17. Umeda, K.; Hase, A.; Matsuo, M.; Horimoto, T.; Ogasawara, J. Prevalence and genetic characterization of cephalosporin-resistant Enterobacteriaceae among dogs and cats in an animal shelter. J. Med. Microbiol. 2019, 68, 339-345. [CrossRef]

18. A Tripartite Guide to Addressing Zoonotic Diseases in Countries Taking a Multisectoral, One Health Approach. Published in 2019 by The Food and Agriculture Organization of the United Nations, The World Organisation for Animal Health and The World Health Organization. Available online: https://www.oie.int/ (accessed on 12 July 2020). 
19. CDC. Prioritizing Zoonotic Diseases for Multisectoral, One Health Collaboration in the United States; CDC: Atlanta, GA, USA, 2019; pp. 5-7.

20. Magiorakos, A.P.; Srinivasan, A.; Carey, R.B.; Carmeli, Y.; Falagas, M.E.; Giske, C.G.; Harbarth, S.; Hindler, J.F.; Kahlmeter, G.; Olsson-Liljequist, B.; et al. Multidrug-resistant, extensively drug-resistant and pandrug-resistant bacteria: An international expert proposal for interim standard definitions for acquired resistance. Clin. Microbiol. Infect. 2012, 18, 268-281. [CrossRef]

21. Joosten, P.; Ceccarelli, D.; Odent, E.; Sarrazin, S.; Graveland, H.; Van Gompel, L.; Battisti, A.; Caprioli, A.; Franco, A.; Wagenaar, J.A.; et al. Antimicrobial usage and resistance in companion animals: A cross-sectional study in three european countries. Antibiotics 2020, 9, 87. [CrossRef]

22. Committee for Medicinal Products for Veterinary use (CVMP) 3 Committee for Medicinal Products for Human Use (CHMP) 2019. Available online: www.ema.europa.eu/contact (accessed on 29 June 2020).

23. Gómez-Poveda, B.; Moreno, M.A. Antimicrobial prescriptions for dogs in the capital of Spain. Front. Vet. Sci. 2018, 5, 309. [CrossRef]

24. Tolun, V.; Küçükbasmaci, Ö.; Törümküney-Akbulut, D.; Çatal, Ç.; Anğ-Küçüker, M.; Anğ, Ö. Relationship between ciprofloxacin resistance and extended-spectrum $\beta$-lactamase production in Escherichia coli and Klebsiella pneumoniae strains. Clin. Microbiol. Infect. 2004, 10, 72-75. [CrossRef]

25. Dupouy, V.; Abdelli, M.; Moyano, G.; Arpaillange, N.; Bibbal, D.; Cadiergues, M.C.; Lopez-Pulin, D.; Sayah-Jeanne, S.; De Gunzburg, J.; Saint-Lu, N.; et al. Prevalence of beta-lactam and quinolone/fluoroquinolone resistance in enterobacteriaceae from dogs in France and Spain-characterization of ESBL/pAmpC isolates, genes, and conjugative plasmids. Front. Vet. Sci. 2019, 6, 279. [CrossRef] [PubMed]

26. Rocha-Gracia, R.C.; Cortés-Cortés, G.; Lozano-Zarain, P.; Bello, F.; Martínez-Laguna, Y.; Torres, C. Faecal Escherichia coli isolates from healthy dogs harbour CTX-M-15 and CMY-2 $\beta$-lactamases. Vet. J. 2015, 203, 315-319. [CrossRef] [PubMed]

27. Clermont, O.; Christenson, J.K.; Denamur, E.; Gordon, D.M. The Clermont Escherichia coli phylo-typing method revisited: Improvement of specificity and detection of new phylo-groups. Environ. Microbiol. Rep. 2013, 5, 58-65. [CrossRef] [PubMed]

28. Vogt, D.; Overesch, G.; Endimiani, A.; Collaud, A.; Thomann, A.; Perreten, V. Occurrence and genetic characteristics of third-generation cephalosporin-resistant Escherichia coli in Swiss retail meat. Microb. Drug Resistance 2014, 20, 485-494. [CrossRef]

29. Dierikx, C.; van der Goot, J.; Fabri, T.; van Essen-Zandbergen, A.; Smith, H.; Mevius, D. Extended-spectrum- $\beta$-lactamase-and AmpC- $\beta$-lactamase-producing Escherichia coli in Dutch broilers and broiler farmers. J. Antimicrob. Chemother. 2013, 68, 60-67. [CrossRef]

30. Valverde, A.; Cantón, R.; Garcillán-Barcia, M.P.; Novais, Â.; Galán, J.C.; Alvarado, A.; De La Cruz, F.; Baquero, F.; Coque, T.M. Spread of bla $a_{\mathrm{CTX}-\mathrm{M}-14}$ is driven mainly by IncK plasmids disseminated among Escherichia coli phylogroups A, B1, and D in Spain. Antimicrob. Agents Chemother. 2009, 53, 5204-5212. [CrossRef]

31. Rumi, M.V.; Mas, J.; Elena, A.; Cerdeira, L.; Muñoz, M.E.; Lincopan, N.; Gentilini, É.R.; Di Conza, J.; Gutkind, G. Co-occurrence of clinically relevant $\beta$-lactamases and MCR-1 encoding genes in Escherichia coli from companion animals in Argentina. Vet. Microbiol. 2019, 230, 228-234. [CrossRef]

32. Jamborova, I.; Dolejska, M.; Vojtech, J.; Guenther, S.; Uricariu, R.; Drozdowska, J.; Papousek, I.; Pasekova, K.; Meissner, W.; Hordowski, J.; et al. Plasmid-mediated resistance to cephalosporins and fluoroquinolones in various Escherichia coli sequence types isolated from rooks wintering in Europe. Appl. Environ. Microbiol. 2015, 81, 648-657. [CrossRef]

33. Börjesson, S.; Egervärn, M.; Lindblad, M.; Englunda, S. Frequent occurrence of extended-spectrum beta-lactamase- and transferable AMPC beta-lactamase-producing Escherichia coli on domestic chicken meat in Sweden. Appl. Environ. Microbiol. 2013, 79, 2463-2466. [CrossRef]

34. Díaz-Jiménez, D.; García-Meniño, I.; Fernández, J.; García, V.; Mora, A. Chicken and turkey meat: Consumer exposure to multidrug-resistant Enterobacteriaceae including mcr-carriers, uropathogenic E. coli and high-risk lineages such as ST131. Int. J. Food Microbiol. 2020, 331, 108750. [CrossRef]

35. Riley, L.W. Extraintestinal foodborne pathogens. Annu. Rev. Food Sci. Technol. 2020, 11, 275-294. [CrossRef] [PubMed] 
36. Díaz-Jiménez, D.; García-Meniño, I.; Herrera, A.; García, V.; López-Beceiro, A.; Alonso, M.P.; Blanco, J.; Mora, A. Genomic characterization of Escherichia coli isolates belonging to a new hybrid aepec/expec pathotype o153:H10-a-st10 eae-beta1 occurred in meat, poultry, wildlife and human diarrheagenic samples. Antibiotics 2020, 9, 192. [CrossRef]

37. Flament-Simon, S.C.; Nicolas-Chanoine, M.H.; García, V.; Duprilot, M.; Mayer, N.; Alonso, M.P.; García-Meniño, I.; Blanco, J.E.; Blanco, M.; Blanco, J. Clonal structure, virulence factor-encoding genes and antibiotic resistance of Escherichia coli, causing urinary tract infections and other extraintestinal infections in humans in spain and france during 2016. Antibiotics 2020, 9, 161. [CrossRef] [PubMed]

38. Flament-Simon, S.C.; García, V.; Duprilot, M.; Mayer, N.; Alonso, M.P.; García-Meniño, I.; Blanco, J.E.; Blanco, M.; Nicolas-Chanoine, M.H.; Blanco, J. High prevalence of ST131 subclades C2-H30Rx and C1-M27 among extended-spectrum $\beta$-lactamase-producing Escherichia coli causing human extraintestinal infections in patients from two hospitals of Spain and France during 2015. Front. Cell. Inf. Microbiol. 2020, 10, 125. [CrossRef] [PubMed]

39. Ferreira, J.C.; Penha Filho, R.A.C.; Andrade, L.N.; Berchieri, A.; Darini, A.L.C. Detection of chromosomal blaCTX-M-2 in diverse Escherichia coli isolates from healthy broiler chickens. Clin. Microbiol. Inf. 2014, 20, O623-O626. [CrossRef] [PubMed]

40. Wang, J.; Huang, X.-Y.; Xia, Y.-B.; Guo, Z.-W.; Ma, Z.-B.; Yi, M.-Y.; Lv, L.-C.; Lu, P.-L.; Yan, J.-C.; Huang, J.-W.; et al. Clonal spread of Escherichia coli ST93 carrying mcr-1-Harboring IncN1-IncHI2/ST3 plasmid among companion animals, China. Front. Microbiol. 2018, 9, 2989. [CrossRef] [PubMed]

41. Zhang, P.; Wang, J.; Wang, X.; Bai, X.; Ma, J.; Dang, R.; Xiong, Y.; Fanning, S.; Bai, L.; Yang, Z. Characterization of five Escherichia coli isolates co-expressing ESBL and mcr-1 resistance mechanisms from different origins in China. Front. Microbiol. 2019, 10, 1994. [CrossRef]

42. Papa-Ezdra, R.; Grill Diaz, F.; Vieytes, M.; García-Fulgueiras, V.; Caiata, L.; Ávila, P.; Brasesco, M.; Christophersen, I.; Cordeiro, N.F.; Algorta, G.; et al. First three Escherichia coli isolates harbouring mcr-1 in Uruguay. J. Glob. Antimicrob. Resistance 2020, 20, 187-190. [CrossRef]

43. Dohmen, W.; Bonten, M.J.M.; Bos, M.E.H.; van Marm, S.; Scharringa, J.; Wagenaar, J.A.; Heederik, D.J.J. Carriage of extended-spectrum $\beta$-lactamases in pig farmers is associated with occurrence in pigs. Clin. Microbiol. Infect. 2015, 21, 917-923. [CrossRef]

44. Hayashi, W.; Tanaka, H.; Taniguchi, Y.; Iimura, M.; Soga, E.; Kubo, R.; Matsuo, N.; Kawamura, K.; Arakawa, Y.; Nagano, Y.; et al. Acquisition of mcr-1 and Cocarriage of Virulence Genes in Avian Pathogenic Escherichia coli Isolates from Municipal Wastewater Influents in Japan. Appl. Environ. Microbiol. 2019, 85. [CrossRef]

45. Johnson, J.R.; Johnston, B.D.; Gordon, D.M. Rapid and specific detection of the Escherichia coli sequence type 648 complex within phylogroup F. J. Clin. Microbiol. 2017, 55, 1116-1121. [CrossRef] [PubMed]

46. Holt, K.E.; Wertheim, H.; Zadoks, R.N.; Baker, S.; Whitehouse, C.A.; Dance, D.; Jenney, A.; Connor, T.R.; Hsu, L.Y.; Severin, J.; et al. Genomic analysis of diversity, population structure, virulence, and antimicrobial resistance in Klebsiella pneumoniae, an urgent threat to public health. Proc. Nat. Acad. Sci. USA 2015, 112, E3574-E3581. [CrossRef] [PubMed]

47. Ludden, C.; Moradigaravand, D.; Jamrozy, D.; Gouliouris, T.; Blane, B.; Naydenova, P.; Hernandez-Garcia, J.; Wood, P.; Hadjirin, N.; Radakovic, M. A one health study of the genetic relatedness of Klebsiella pneumoniae and their mobile elements in the east of England. Clin. Infect. Dis. 2020, 70, 219-226. [CrossRef] [PubMed]

48. Domokos, J.; Damjanova, I.; Kristof, K.; Ligeti, B.; Kocsis, B.; Szabo, D. Multiple benefits of plasmid-mediated quinolone resistance determinants in Klebsiella pneumoniae ST11 high-risk clone and recently emerging ST307 clone. Front. Microbiol. 2019, 10, 157. [CrossRef]

49. Villa, L.; Feudi, C.; Fortini, D.; Brisse, S.; Passet, V.; Bonura, C.; Endimiani, A.; Mammina, C.; Ocampo, A.M.; Jimenez, J.N.; et al. Diversity, virulence, and antimicrobial resistance of the KPCproducing Klebsiella pneumoniae ST307 clone. Microb. Genom. 2017, 3, e000110. [CrossRef]

50. Carvalho, I.; Alonso, C.A.; Silva, V.; Pimenta, P.; Cunha, R.; Martins, C.; Igrejas, G.; Torres, C.; Poeta, P. Extended-spectrum beta-lactamase-producing Klebsiella pneumoniae Isolated from healthy and sick dogs in Portugal. Microb. Drug Resistance 2019, 26, 709-715. [CrossRef]

51. Breakpoint Tables for Interpretation of MICs and Zone Diameters. Version 10.0, valid from 2020-01-01. European Committee on Antimicrobial Susceptibility Testing. Available online: http://www.eucast.org/ clinical_breakpoints/ (accessed on 12 July 2020). 
52. García-Meniño, I.; García, V.; Mora, A.; Díaz-Jiménez, D.; Flament-Simon, S.C.; Alonso, M.P.; Blanco, J.E.; Blanco, M.; Blanco, J. Swine enteric colibacillosis in Spain: Pathogenic potential of mcr-1 ST10 and ST131 E. Coli Isolates. Front. Microbiol. 2018, 9, 2659. [CrossRef]

53. Johnson, J.R.; Porter, S.; Johnston, B.; Kuskowski, M.A.; Spurbeck, R.R.; Mobley, H.L.T.; Williamson, D.A. Host Characteristics and Bacterial Traits Predict Experimental Virulence for Escherichia coli Bloodstream Isolates from Patients with Urosepsis; Oxford University Press: Oxford, UK, 2015. [CrossRef]

54. Spurbeck, R.R.; Dinh, P.C.; Walk, S.T.; Stapleton, A.E.; Hooton, T.M.; Nolan, L.K.; Kim, K.S.; Johnson, J.R.; Mobley, H.L.T. Escherichia coli isolates that carry vat, fyua, chua, and yfcv efficiently colonize the urinary tract. Infect. Immun. 2012, 80, 4115-4122. [CrossRef]

55. Guinée, P.A.; Jansen, W.H.; Maas, H.M.; le Minor, L.; Beaud, R. An unusual H antigen (Z66) in strains of Salmonella typhi. Ann. Microbiol. 1981, 132, 331-334. Available online: http://www.ncbi.nlm.nih.gov/pubmed/ 7294611 (accessed on 7 June 2020).

56. Weissman, S.J.; Johnson, J.R.; Tchesnokova, V.; Billig, M.; Dykhuizen, D.; Riddell, K.; Rogers, P.; Qin, X.; Butler-Wu, S.; Cookson, B.T.; et al. High-resolution two-locus clonal typing of extraintestinal pathogenic Escherichia coli. Appl. Environ. Microbiol. 2012, 78, 1353-1360. [CrossRef]

57. Wirth, T.; Falush, D.; Lan, R.; Colles, F.; Mensa, P.; Wieler, L.H.; Karch, H.; Reeves, P.R.; Maiden, M.C.J.; Ochman, H.; et al. Sex and virulence in Escherichia coli: An evolutionary perspective. Mol. Microbiol. 2006, 60, 1136-1151. [CrossRef] [PubMed]

58. Diancourt, L.; Passet, V.; Verhoef, J.; Grimont, P.A.D.; Brisse, S. Multilocus sequence typing of Klebsiella pneumoniae nosocomial isolates. J. Clinc. Microbiol. 2005, 43, 4178-4182. [CrossRef] [PubMed]

(C) 2020 by the authors. Licensee MDPI, Basel, Switzerland. This article is an open access article distributed under the terms and conditions of the Creative Commons Attribution (CC BY) license (http://creativecommons.org/licenses/by/4.0/). 